\title{
Vibration Data Processing for Bedload Monitoring in Underwater Environments ${ }^{\dagger}$
}

\author{
Gabriel Vasile \\ Grenoble-Image-sPeach-Signal-Automatics Lab, CNRS/Grenoble INP, Université Grenoble Alpes, \\ 38000 Grenoble, France; gabriel.vasile@grenoble-inp.fr; Tel.: +33-(0)476-826-334 \\ + This paper is an extended version of our paper published in MTS/IEEE North American \\ OCEANS conference.
}

Received: 10 July 2020; Accepted: 25 August 2020; Published: 28 August 2020

check for updates

\begin{abstract}
This paper proposes a novel data processing framework dedicated to bedload monitoring in underwater environments. After calibration, by integration the of total energy in the nominal bandwidth, the proposed experimental set-up is able to accurately measure the mass of individual sediments hitting the steel plate. This requires a priori knowledge of the vibration transients in order to match a predefined dictionary. Based on unsupervised hierarchical agglomeration of complex vibration spectra, the proposed algorithms allow accurate localization of the transients corresponding to the shocks created by sediment impacts on a steel plate.
\end{abstract}

Keywords: hierarchical agglomeration; complex vibration spectra; bedload monitoring; underwater acoustics

\section{Introduction}

Underwater bedload transport surveys are important for assessing stability issues such as reservoir silting or channel self-cleaning. To this purpose, sediment traps are currently used to derive the sediment balances.

Monitoring of the bedload transport in underwater environments is crucial for understanding stability issues. The Birkbeck sampler has become one of the preferred methods for in situ bedload measurements [1]. Being an integral measure of the transported sediment volume in a certain time interval, this direct method suffers from one main drawback: it has a finite capacity. Without the use of devices such as a sludge pump to empty the accumulating sediment, the sampler fills and eventually ceases to yield data. Furthermore, bedload transport in rivers can be extrapolated using empirical models such as the one used by Radecki-Pawlik et al. [2] for the Mlynne and Lososina Streams (Polish Carpathians). An extensive review on water-worked gravel bed natural evolution can be found in [3].

An alternative nonintrusive bedload monitoring instrument is the buried geophone station [4-6]. Under protection of steel plates, several geophones can provide continuous and automatic measurements, even during large floods. Every stone passing the steel geophone-equipped plate generates an impulse recorded by the signal acquisition board. Using a calibrated voltage thresholding scheme, the grain impacts are recorded and counted. One can notice that this instrument is highly sensitive to the environmental noise, as it operates at low frequencies $(<1 \mathrm{KHz})$.

In [7], Bogen and Moen used piezoelectric acoustic transducers for bedload monitoring stations in Norway. The reported results present a clear correspondence with the sediment flow, but no estimation scheme is proposed.

Very recently, Antoniazza et al. proposed an extensive study on the variability of bedload transport monitoring by the Swiss plate geophone system [8]. One of the conclusions was that, after calibration, sediment grain-size identification can be achieved by matching particle size classes [9]. 
In this paper, the potential of ultrasound (US) transducers coupled with piezoelectric accelerometers for bedload monitoring stations is further explored in a controlled laboratory environment. The obtained results provide interesting sensitivity capabilities, in accordance with the ground truth. The main objectives are to validate the operating frequency of the transducers, to provide consistent calibration and processing, and to evaluate the uncertainty of the derived measurements. To pursue these objectives, an experimental platform, illustrated in Figure 1a, has been developed and tested in the GIPSA-Lab controlled tank system.

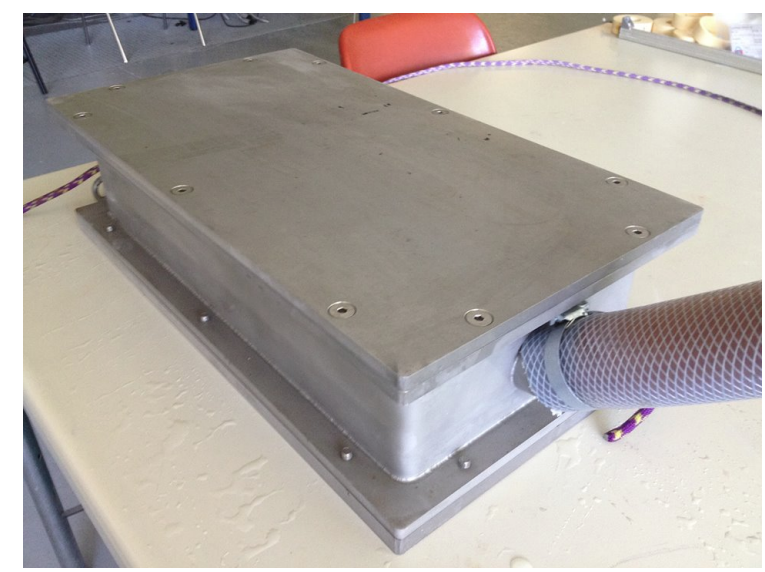

(a)

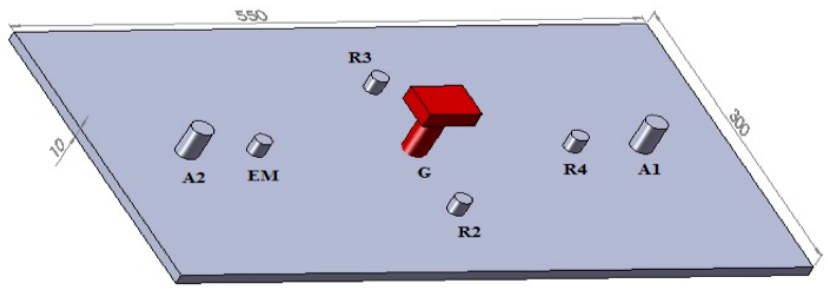

(b)

Figure 1. Multisensor bedload transport monitoring platform: (a) general view and (b) set-up of the acoustic sensors on the top steel plate (units expressed in $\mathrm{cm}$ ).

The following sensors have been employed.

- Four ultrasound contact transducers PAC R50 (EM, R2, R3, and R4).

- Two calibrated piezoelectric accelerometers Endevco 233E (A1 and A1).

- One geophone R.T. Clark (G).

The EM US transducer has been excited using the ENI 2100L power amplifier and the Picotest G5100A arbitrary signal generator. The received signals from A1, A2, R3, and R4 have been conditioned using the Nexus low noise amplifier and recorded using the PXIe-1082 NI system.

After calibration, by integrating the total energy in the nominal bandwidth, the proposed experimental setup is able to accurately measure the mass of individual sediments hitting the steel plate. This requires a priori knowledge of the vibration transients in order to match a predefined dictionary. We propose a novel framework for detecting and localizing the transients (in the passive configuration) corresponding to the shocks created by sediment impacts on the steel plate. The proposed algorithm is based on a hierarchical agglomeration of complex vibration spectra. The multivariate segmentation algorithm proposed in [10] is selected: the multimodal signals are analyzed by exploiting the asymptotic distribution of the covariance matrix of the complex spectra.

Within this context, this manuscript synthesizes the results presented in $[11,12]$ in order to propose a unified acoustic data processing and analysis framework for the monitoring of the bedload transport in underwater environments. The paper is structured as follows. Section 2 introduces the measurement 
methodology, Section 3 describes the proposed data processing framework, and Section 4 presents some qualitative and quantitative performance assessment. Section 5 concludes the paper.

\section{Calibration and Measurement}

For the proposed bedload monitoring system, the static calibration is a direct relationship between the output and the input of the system determined experimentally.

Static calibration requires a reference standard, to which the output is reported. For our system of sensors, the calibration process determines a relationship between the input voltage controlling the EM US transducer and the output characteristic, called static characteristic curve or calibration. Figure 2 shows the static characteristic in water, measured by transmitting a sinusoidal signal and looking at the reception of the peak value of the Fourier transform. The value obtained supports the idea that our system is linear in all cases. In circumstances where the assumption of stationary is unsustainable, linear dynamic models need to be replaced by more sophisticated models.

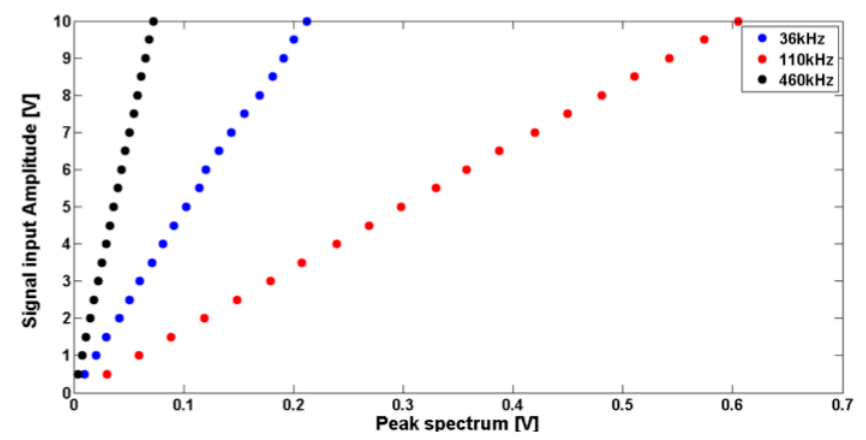

Figure 2. Multisensor bedload monitoring platform: static characteristics.

The dynamic characteristic of each sensor is related to the performance when the input EM voltage signal is a function of time. The speed of response of a system refers to the ability to respond to sudden changes in the amplitude of the input signal. Thus, the response rate is calculated based on the parameters describing the system performance under the transition: constant measuring of the time delay (latency measurement), set up time (settling time), the dead time, and range dynamics. The obtained dynamic characteristics are illustrated in Figure 3 and match the previously derived static characteristics.

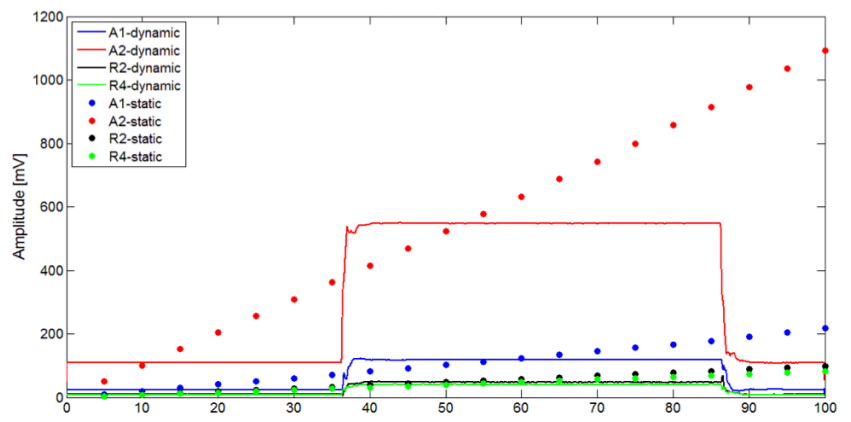

Figure 3. Multisensor bedload monitoring platform: dynamic characteristics: received signal (mV) vs. time (ms).

The transfer function of an acoustic transducer can be computed by positioning two transducer elements in transmission configuration:

- by continuous excitation of one transducer with wideband white Gaussian noise the other responds in its nominal frequency band;

- by considering the performance parameters from the dynamic characteristic, one can define an impulsive excitation for estimating the system transfer function. Accordingly, by taking 
the impulse duration (short sine waveform) to be smaller than the system stabilization time, the transducer is sensing this calibration signal as an impulse and not as a step function.

In this manuscript, the second method for estimating the transfer function is employed and the results are illustrated in Figure 4.

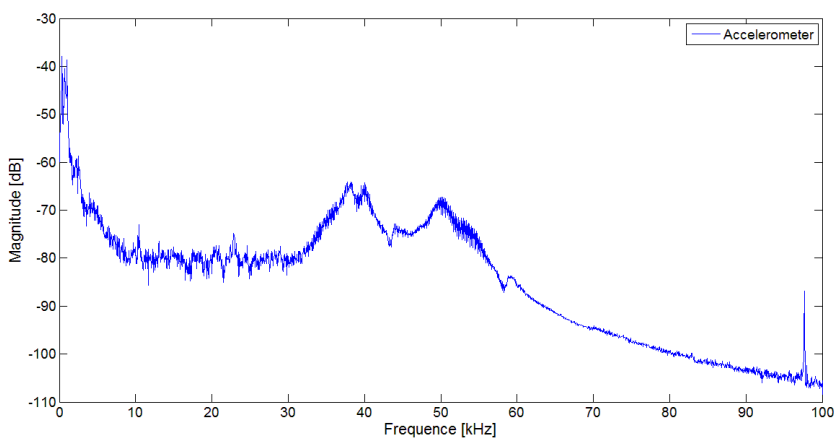

(a)

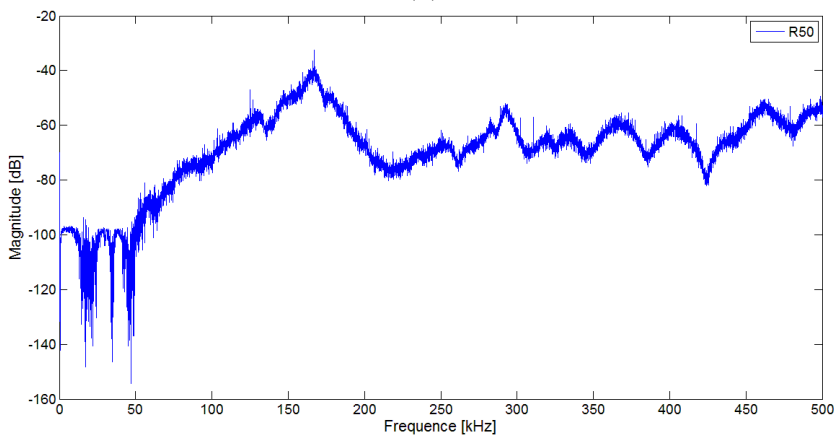

(b)

Figure 4. Transducer transfer function: (a) accelerometer and (b) ultrasound transducer.

After using controlled steel balls for calibration, two types of sediments were investigated and confidence intervals for the mass measurements have been obtained. Notice that the maximum speed in water has been reached before hitting the multisensor bedload monitoring platform. Figure 5 shows the obtained bedload mass measurements in a passive configuration in the nominal bandwidth of the impact $(<14 \mathrm{KHz})$. One can observe that the bedload monitoring platform can provide competitive results, both in terms of measurement error and confidence intervals in controlled noise free environment.
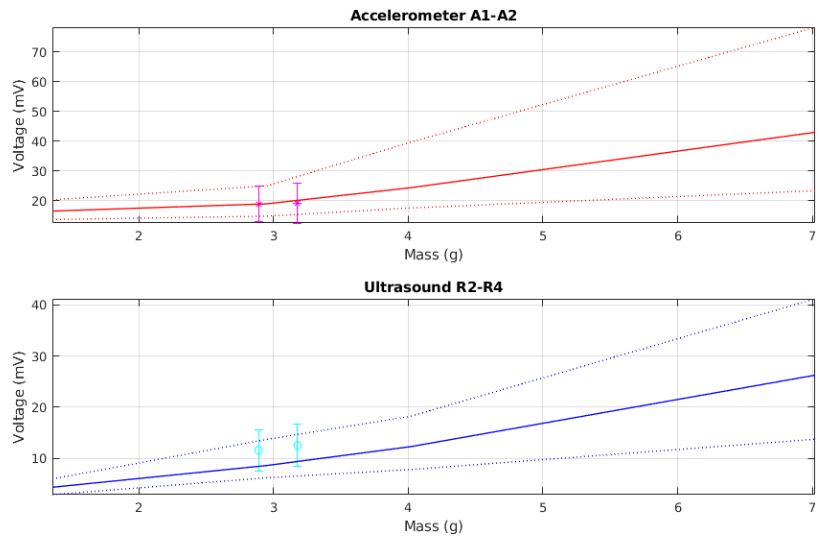

(a)

Figure 5. Cont. 


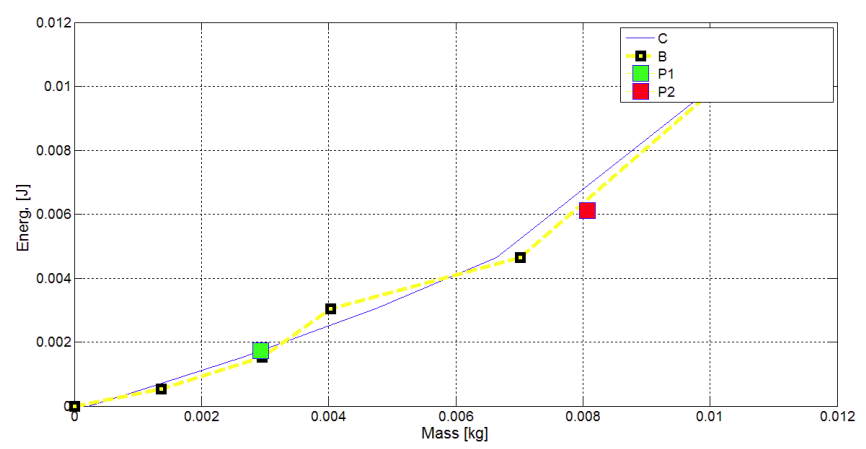

(b)

Figure 5. Bedload mass measurements with passive configuration in the nominal bandwidth of the impact ( $<14 \mathrm{KHz}$ ): (a) for each transducer type and (b) aggregated; B—steel balls in yellow, P1—3 g rock in green, $\mathrm{P} 2-8 \mathrm{~g}$ rock in red, $\mathrm{C}-2$ nd-order polynomial fitting in blue.

\section{Hierarchical Agglomeration of Complex Vibration Spectra}

In order to correctly employ the measurement system presented in Section 2, reliable a priori knowledge of the vibration transients is required in order to match the predefined calibration dictionary. Thus, a preliminary transient segmentation step is required for the recorded acoustic data.

The first stage of the proposed segmentation scheme is preprocessing. As each acquisition is triggered on the amplitude level of the sensor exhibiting the fastest response time (the accelerometers in our case), the obtained transient signal is not symmetric. In order to improve the spectral representation, each received signal is circularly shifted to the right and filtered in the common bandwidth. The complex spectrum is obtained by applying the fast Fourier transform (FFT) on each signal. One advantage of this representation resides in the fact that it is independent of the time of arrival of each transient.

\subsection{SIRV Spectral Estimation}

In the next step, a multivariate random vector is obtained by concatenating all the complex spectra of the available signals. For each sensor, let $k$ be the $m \times 1$ complex target vector corresponding to the same frequency range. One way to model the statistical properties of this multivariate random vector leads to Spherically Invariant Random Vectors (SIRV) [13]. SIRV are defined as the product of a square root of a positive random variable $\tau$ (variations in power) with an independent circular complex Gaussian vector $\mathbf{z}$ with zero mean and covariance matrix $[M]=E\left\{\mathbf{z z}^{H}\right\}$ (Gaussian kernel):

$$
\mathbf{k}=\sqrt{\tau} \mathbf{z}
$$

where the superscript $H$ denotes the complex conjugate transposition and $E\{\cdot\}$ is the mathematical expectation. SIRV representation is not unique, so a normalization condition is necessary. Indeed, if $\left[M_{1}\right]$ and $\left[M_{2}\right]$ are two covariances matrices such that $\left[M_{1}\right]=\alpha\left[M_{2}\right]$, then $\left\{\tau_{1},\left[M_{1}\right]\right\}$ and $\left\{\tau_{2}=\tau_{1} / \alpha,\left[M_{2}\right]\right\}$ describe the same SIRV. In this manuscript, the trace of the covariance matrix is normalized to $p$ the dimension of target complex vector [13].

For $N$ independent and identically distributed (i.i.d.) data, the likelihood function to maximize with respect to $[M]$ and $\tau_{i}$, is given by

$$
L_{\mathbf{k}}\left(\mathbf{k}_{1}, \ldots, \mathbf{k}_{N} ;[M], \tau_{1}, \ldots, \tau_{N}\right)=\frac{1}{\pi^{m N} \operatorname{det}\{[M]\}^{N}} \times \prod_{i=1}^{N} \frac{1}{\tau_{i}^{m}} \exp \left(-\frac{\mathbf{k}_{i}^{\dagger}[M]^{-1} \mathbf{k}_{i}}{\tau_{i}}\right) .
$$


For a given $[\mathrm{M}]$, maximizing $L_{\mathbf{k}}\left(\mathbf{k}_{1}, \ldots, \mathbf{k}_{N} ;[M], \tau_{1}, \ldots, \tau_{N}\right)$ with respect to $\tau_{i}$ yields the texture ML estimator

$$
\widehat{\tau}_{i}=\frac{\mathbf{k}_{i}^{\dagger}[M]^{-1} \mathbf{k}_{i}}{m} \text {. }
$$

Replacing $\tau_{i}$ in the SIRV likelihood function by their ML estimates, the generalized likelihood is obtained as

$$
L_{\mathbf{k}}^{\prime}\left(\mathbf{k}_{1}, \ldots, \mathbf{k}_{N} ;[M]\right)=\frac{1}{\pi^{m N} \operatorname{det}\{[M]\}^{N}} \times \prod_{i=1}^{N} \frac{m^{m} \exp (-m)}{\left(\mathbf{k}_{i}^{\dagger}[M]^{-1} \mathbf{k}_{i}\right)^{m}} .
$$

The ML estimator of the normalized covariance matrix in the deterministic texture case is obtained by canceling the gradient of $L_{\mathbf{k}}^{\prime}$ with respect to $[M]$ as the solution of the following recursive equation,

$$
[\widehat{M}]_{F P}=f\left([\widehat{M}]_{F P}\right)=\frac{m}{N} \sum_{i=1}^{N} \frac{\mathbf{k}_{i} \mathbf{k}_{i}^{\dagger}}{\mathbf{k}_{i}^{\dagger}[\widehat{M}]_{F P}^{-1} \mathbf{k}_{i}}=\frac{m}{N} \sum_{i=1}^{N} \frac{\mathbf{z}_{i} \mathbf{z}_{i}^{\dagger}}{\mathbf{z}_{i}^{\dagger}[\widehat{M}]_{F P}^{-1} \mathbf{z}_{i}} .
$$

This approach has been used in [14] by Conte et al. to derive a recursive algorithm for estimating the matrix $[M]$. This algorithm consists in computing the fixed point of $f$ using the sequence $\left([M]_{i}\right)_{i \geq 0}$ defined by

$$
[M]_{i+1}=f\left([M]_{i}\right) .
$$

Its asymptotic distribution can be assimilated to the Wishart Probability Density Function (PDF) [13].

This study has been completed by the work of Pascal et al. $[15,16]$, which established the existence and the uniqueness, up to a scalar factor, of the Fixed Point estimator of the normalized covariance matrix, as well as the convergence of the recursive algorithm whatever the initialization. The algorithm can therefore be initialized with the identity matrix $[M]_{0}=\left[I_{m}\right]$. One way to analyze the convergence of the Fixed Point estimator consists in evaluating the following criterion,

$$
C(i)=\frac{\|[\widehat{M}](i+1)-[\widehat{M}](i)\|_{F}}{\|[\widehat{M}](i)\|_{F}},
$$

where $\|. . .\|_{F}$ represents the Frobenius norm. When computing the FP estimator, Equation (6) is iterated until $C$ becomes smaller than a predefined lower limit. Note that only few iterations suffice to reach an error less than $10^{-15}$ [15].

In this way, each random vector is described by its normalized covariance matrix, which is independent on the total power at the reception. This will form the feature space for the transient segmentation algorithm.

\subsection{Hierarchical Segmentation}

The hierarchical segmentation algorithm from [10] is adapted to the vector of complex spectra. The segmentation strategy is a classical iterative merge algorithm. At each iteration, the two segments which minimize the Stepwise Criterion (SC) are merged. Illustrated in Figure 6, the basic principle of the proposed hierarchical segmentation can be divided into three steps :

1. Definition of an initial partition (which is formed by the acquired buffers, in our case).

2. For each segments pair, SC is computed (see Section 3.3). Then, the two segments which minimize the criterion are found and merged.

3. Stop if the maximum number of merges is reached, otherwise go to step 2. The stop criterion is discussed in Section 3.4. 


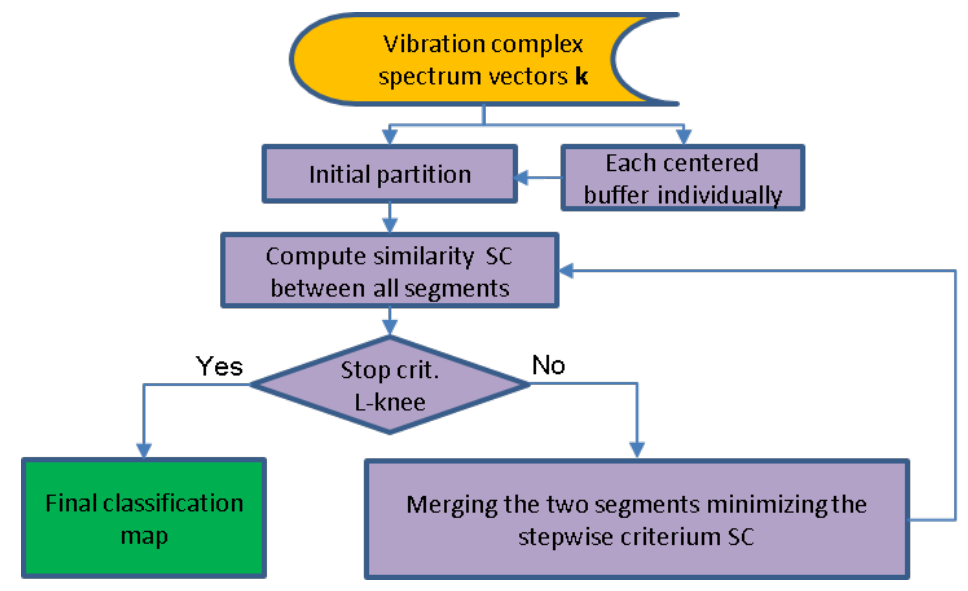

Figure 6. Flowchart of the proposed hierarchical segmentation algorithm.

\subsection{Similarity Measure}

At each iteration, merging two segments yields a decrease in the log-likelihood function. The stepwise criterion is based on this consideration. The hierarchical segmentation algorithm merges the two segments $S_{i}$ and $S_{j}$, which minimizes the loss of likelihood of the partition (which is defined as the sum of likelihoods of partition's segments). The stepwise criterion $\left(\mathrm{SC}_{i, j}\right)$ can be expressed as

$$
\mathrm{SC}_{i, j}=\operatorname{MLL}\left(S_{i}\right)+\operatorname{MLL}\left(S_{j}\right)-\operatorname{MLL}\left(S_{i} \cup S_{j}\right),
$$

where $\operatorname{MLL}(\cdot)$ denotes the segment maximum log-likelihood function; it is the log-likelihood of the segment (samples in each segment are considered independent realizations) with respect to the assumed probability density function (the Wishart distribution in our case) whose parameters are estimated in the maximum likelihood (thus, the name) sense. Its expression is given by

$$
\operatorname{MLL}(S)=\sum_{i \in S} \ln \left(p_{\mathbf{k}}\left(\mathbf{k}_{i} \mid \theta_{S}\right)\right)
$$

$\theta_{S}$ represents the set of distribution parameters (normalized covariance matrix in our case).

Generalized Maximum Log-Likelihood (GMLL)

In general, the normalized covariance matrix is unknown. One solution consists in replacing the SIRV parameters by their estimates. After replacing the covariance matrix $[M]$ by its respective ML estimators, the stepwise criterion becomes

$$
\mathrm{SC}_{i, j}=\operatorname{GMLL}\left(S_{i}\right)+\operatorname{GMLL}\left(S_{j}\right)-\operatorname{GMLL}\left(S_{i} \cup S_{j}\right),
$$

where GMLL $(S)$ is the generalized maximum log-likelihood function for segment $S$.

For the Wishart PDF, the generalized maximum log-likelihood function for segment $S$ is

$$
\operatorname{GMLL}(S)=-p N \ln (\pi)-N \ln \left\{\left|\left[\hat{M}_{M L}\right]\right|\right\}
$$

where $\left[\hat{M}_{M L}\right]$ is the ML estimator of $\left[M_{M L}\right]$ for segment $S$ (5).

\subsection{Stop Criterion}

The use of the L-method [17] has been considered in this paper. This method employs the very same error (quality) function that is used to perform cluster merging during the hierarchical segmentation algorithm, specifically the Log-Likelihood Function (LLF) of the partition (i.e., the sum of the MLL values for all the segments of the partition). As this is readily computed during the proposed 
method, no further computational effort is required. The knee of this error function is identified and the optimal number of clusters is chosen at that point. The knee of a curve is somewhat similar to the point of maximum curvature.

As detailed in [10], the hierarchical segmentation algorithm is generally suboptimal as neither the initial partition nor the iterative merging of the segments is guaranteed to be optimal in the general sense, although the iterative merging is still the optimal processing for the imposed initial partitioning and a segment neighboring condition. The L-method divides the graph of the error function into two parts and approximates each part with a straight line. The pair of lines that most closely fit the error function curve is retained, and their junction point is the knee. The best pair of lines is that which minimizes the weighted sum of approximation errors for the two parts of the error function graph. One can refer to the work in [10] for a more in-depth analysis.

\section{Results and Discussion}

The proposed experimentations were carried in the GIPSA-Lab controlled tank system. Figures 7 and 8 illustrate the transient signals obtained from the four sensors (accelerometers and ultrasound) in passive configuration, after right circular shift and common bandpass filtering.

In order to build the testing database, five steel balls with different diameters have been selected for the experiments. For each ball, 25 independent impacts have been recorded using the bedload transport monitoring platform from Figure 1. The balls have been manually released form the surface of the water at approximately the same position on the steel plate. An independent buffer has been recorded for each impact with the four sensors simultaneously and coherently.

After concatenating the $25 \times 5$ buffers, Figure 9 shows the derived signals used to test the proposed hierarchical agglomeration algorithm.

The obtained results are presented in the confusion matrix from Table 1. There are at least two facts to be noticed. First, the diagonal structure of the confusion matrix reveals a rather good classification accuracy. Second, the L-method stopped the segment merging at eight classes. This is explained by the fact that the impacts of the ball B4 have been split in two classes, while some impacts from B1 and B3 generated two additional classes. This is in agreement with the subjective visual assessment of the signals from Figure 9.

For comparison purposes, the conventional K-means classifier has been applied on the same dataset with the number of classes equal to 8 (as before). The input feature vector is formed by taking the trace of the sample covariance matrix estimated using the complex spectra within each buffer. The resulting confusion matrix is presented in Table 2. One can observe that class TC 4 cannot be retrieved in this case.

Finally, an overall quantitative indicator of the performance of the proposed algorithm is the classification accuracy $A$, defined as

$$
A=\frac{\sum_{i=0}^{5} T P_{B i}+\sum_{i=0}^{5} T N_{B i}}{5 T} \times 100,
$$

with $T P_{B i}$ the true positive for the steel ball $B i, T N_{B i}$ the true negative, and $T=125$ the total population. After fusing the classes SC 5 and SC 6, SC 2 and SC 8, and SC 4 and SC 7, we can compute the total classification accuracy according to Equation (12).

The obtained value is $A_{H}=90.9 \%$ for the proposed hierarchical agglomeration algorithm, while $A_{K}=71.9 \%$ for the conventional K-means classifier. 
Table 1. Quantitative performance assessment for the proposed hierarchical agglomeration algorithm: confusion matrix. Stepwise criterion (SC) 1-8 are the obtained classes, while TC $1-5$ are the ground truth-based classes.

\begin{tabular}{lccccc}
\hline & TC 1 & TC 2 & TC 3 & TC 4 & TC 5 \\
\hline SC 1 & 25 & 8 & 0 & 0 & 0 \\
\hline SC 2 & 0 & 15 & 6 & 0 & 0 \\
\hline SC 3 & 0 & 0 & 19 & 8 & 0 \\
\hline SC 4 & 0 & 0 & 3 & 13 & 5 \\
\hline SC 5 & 0 & 0 & 0 & 0 & 11 \\
\hline SC 6 & 0 & 0 & 0 & 0 & 9 \\
\hline SC 7 & 0 & 0 & 0 & 4 & 0 \\
\hline SC 8 & 0 & 2 & 0 & 0 & 0 \\
\hline
\end{tabular}

Table 2. Quantitative performance assessment for the K-means algorithm: confusion matrix. SC 1-8 are the obtained classes, while TC 1-5 are the ground truth-based classes.

\begin{tabular}{lccccc}
\hline & TC 1 & TC 2 & TC 3 & TC 4 & TC 5 \\
\hline SC 1 & 5 & 0 & 0 & 0 & 0 \\
\hline SC 2 & 3 & 0 & 0 & 0 & 0 \\
\hline SC 3 & 11 & 0 & 0 & 0 & 0 \\
\hline SC 4 & 6 & 7 & 0 & 0 & 0 \\
\hline SC 5 & 0 & 12 & 1 & 0 & 0 \\
\hline SC 6 & 0 & 6 & 11 & 0 & 0 \\
\hline SC 7 & 0 & 0 & 13 & 4 & 0 \\
\hline SC 8 & 0 & 0 & 0 & 21 & 25 \\
\hline
\end{tabular}
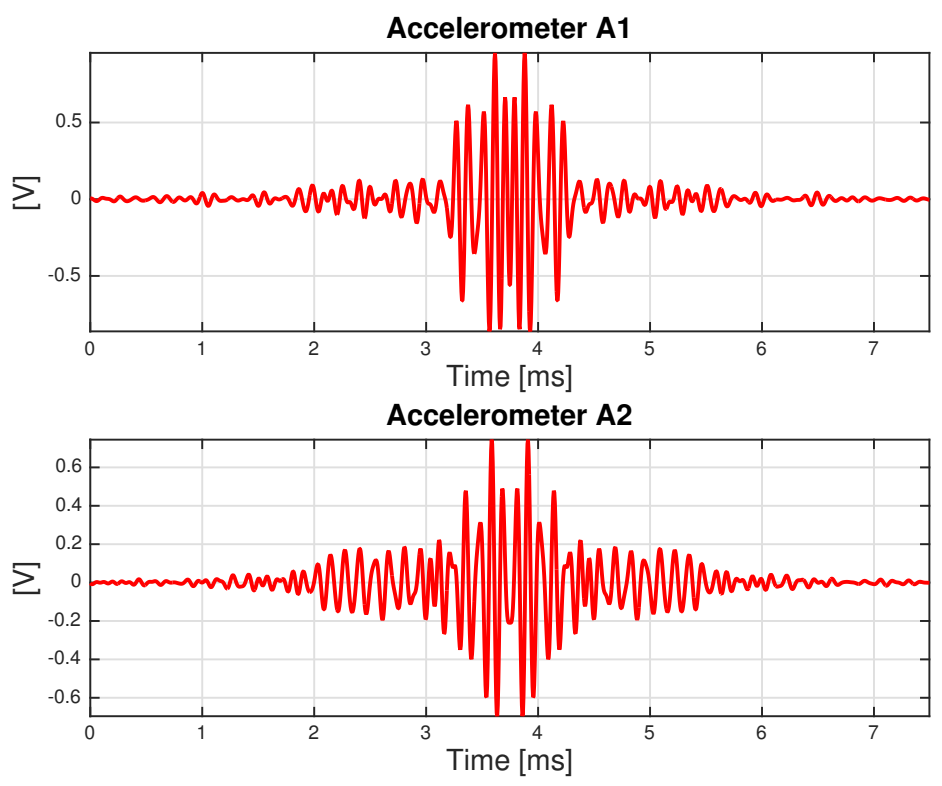

(a)

Figure 7. Cont. 

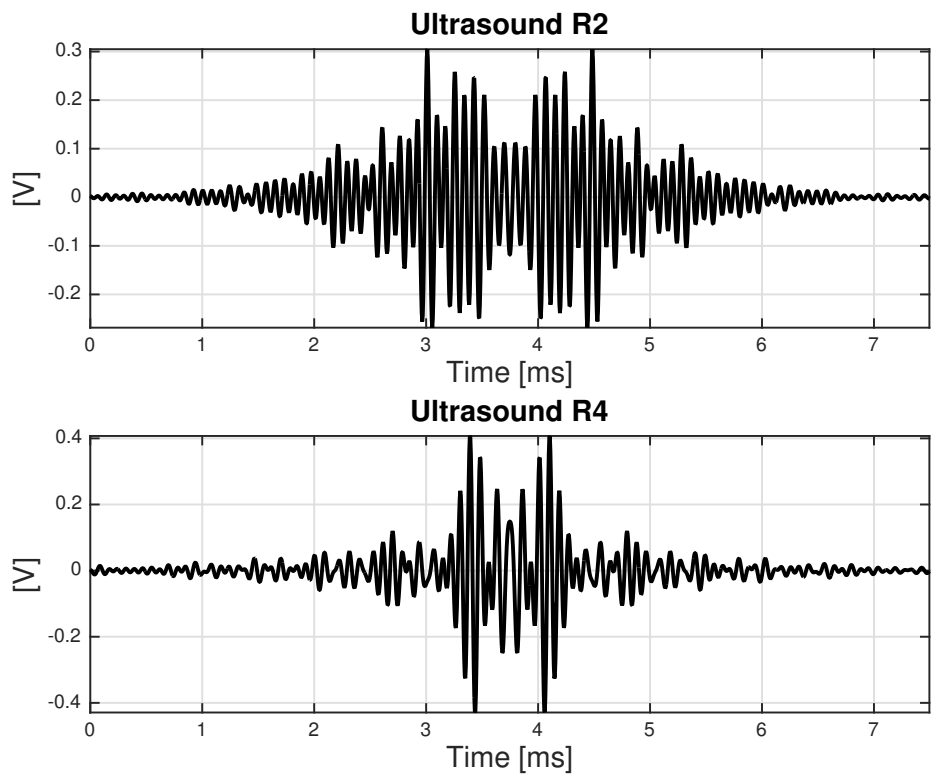

(b)

Figure 7. Sensors recordings in passive configuration, 1 buffer triggered by A1: (a) accelerometers and (b) ultrasound transducers.
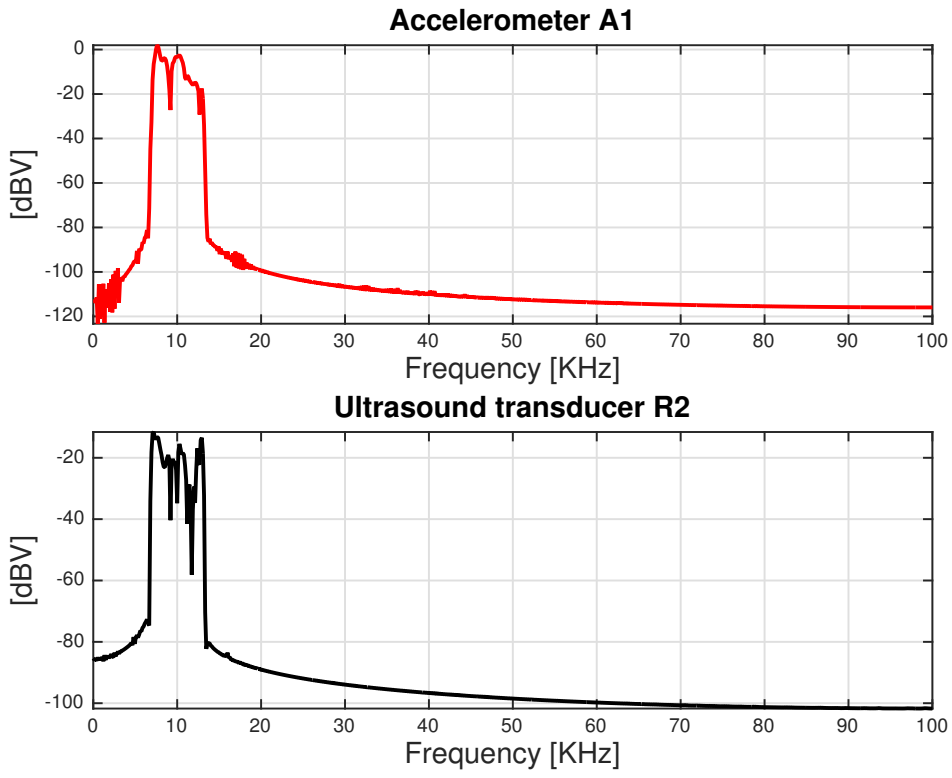

Figure 8. Sensors recordings spectra in passive configuration, 1 buffer triggered by A1: accelerometer A1 (up) and ultrasound transducer R2 (down). 

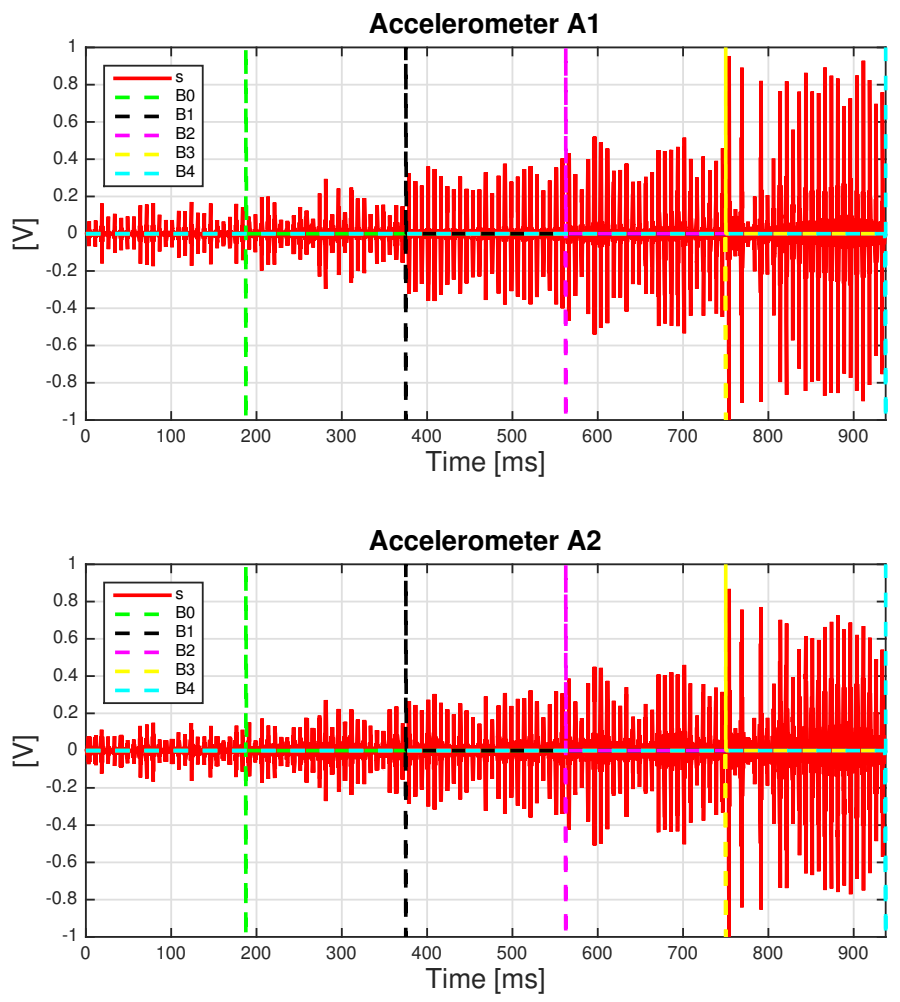

(a)
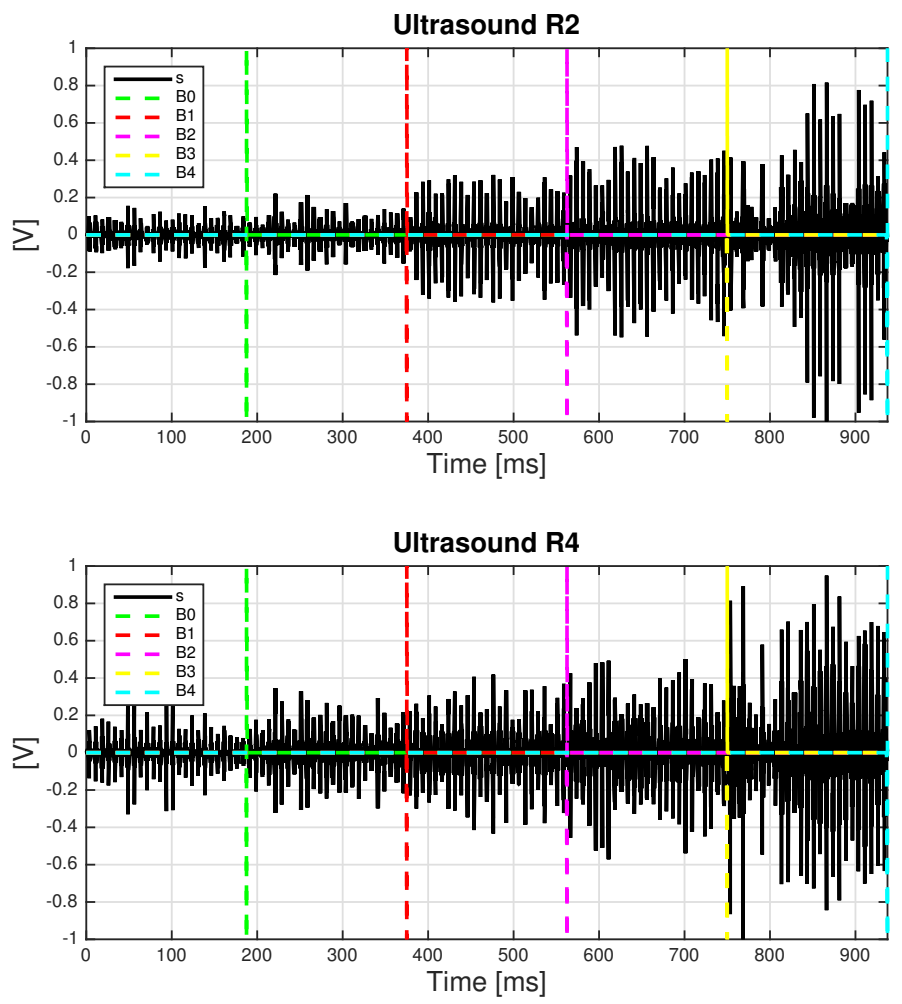

(b)

Figure 9. Sensors recordings in passive configuration, 1 buffer triggered by A1: (a) accelerometers and (b) ultrasound transducers. 


\section{Conclusions}

This paper proposed a new framework for detecting and localizing the transients corresponding to the shocks created by sediment impacts on the steel plate. The proposed classification strategy is based on hierarchical agglomeration of complex vibration spectra: the multimodal signals were analyzed by exploiting the asymptotic distribution (SIRV) of the normalized covariance matrix. Qualitative and quantitative performance assessment has been carried out using vibration signals recorded by the multisensor bedload transport monitoring platform form the GIPSA-Lab.

One limitation of the current classification scheme is the case of multiple sediments hitting the steel plate in the same time. In this scenario, the proposed algorithm will provide an estimation of an equivalent larger impact, only.

Future work will enroll in two main directions. First, we will try to explore as much as possible all the benefits of the proposed classification algorithm in real life scenarios. Second, we will continue with improving the description of transient vibration signals by using non-stationary time-frequency representations instead of the Fourier transform.

Funding: This research was funded by the National Center for Scientific Research (CNRS), France and by the French Electricty Company (EDF), France.

Acknowledgments: The author would like to thank the anonymous reviewers and the associate editor for the helpful comments and suggestions. Thanks go to the French Electricity Company (EDF) for providing part of the hardware used in this study.

Conflicts of Interest: The author declares no conflicts of interest. The funders had no role in the design of the study; in the collection, analyses, or interpretation of data; in the writing of the manuscript; or in the decision to publish the results.

\section{Abbreviations}

The following abbreviations are used in this manuscript.

$\begin{array}{ll}\text { US } & \text { Ultrasound } \\ \text { GIPSA-Lab } & \text { Grenoble-Image-sPeech-Signal-Automatics Lab } \\ \text { FFT } & \text { Fast Fourier Transform } \\ \text { SIRV } & \text { Spherically Invariant Random Vectors } \\ \text { SC } & \text { Stepwise Criterion } \\ \text { ML } & \text { Maximum Likelihood } \\ \text { PDF } & \text { Probability Density Function } \\ \text { LLF } & \text { Log-Likelihood Function }\end{array}$

\section{References}

1. Laronne, J.B.; Alexandrov, Y.; Bergman, N.; Cohen, H.; Garcia, C.; Habersack, H.; Powell, D.M.; Reid, I. The Continuous Monitoring of Bedload Flux in Various Fluvial Environments; International Association of Hydrological Sciences Publication: Wallingford, UK, 2003; Volume 283, pp. 134-145.

2. Radecki-Pawlik, A.; Kubon, P.; Radecki-Pawlik, B.; Plesinski, K. Bed-Load Transport in Two Different-Sized Mountain Catchments: Mlynne and Lososina Streams, Polish Carpathians. Water 2019, 11, 272. [CrossRef]

3. Padhi, E.; Dey, S.; Desai, V.; Gaudio, N.P.R. Water-Worked Gravel Bed: State-of-the-Art Review. Water 2019, 11, 694. [CrossRef]

4. Rickenmann, D.; Turowski, J.M.; Fritschi, B.; Klaiber, A.; Ludwig, A. Bedload transport measurements at the Erlenbach stream with geophones and automated basket samplers. Earth Surf. Process. Landf. 2012, 37, 1000-1011. [CrossRef]

5. Rickenmann, D.; Turowski, J.M.; Fritschi, B.; Wyss, C.; Laronne, J.B.; Barzilai, R.; Reid, I.; Kreisler, A.; Aigner, J.; Seitz, H.; et al. Bedload transport measurements with impact plate geophones: Comparison of sensor calibration in different gravel-bed streams. Earth Surf. Process. Landf. 2014, 39, 928-942. [CrossRef] 
6. Hilldale, R.; Carpenter, W.; Goodwiller, B.; Chambers, J.; Randle, T. Installation of Impact Plates to Continuously Measure Bed Load: Elwha River, Washington, USA. J. Hydraul. Eng. 2015, 141, 06014023. [CrossRef]

7. Bogen, J.; Moen, K. Bedload measurements with a new passive acoustic sensor. In Erosion and Sediment Transport Measurement in Rivers: Technological and Methodological Advances; IAHS Press: Wallingford, UK, 2003; pp. 181-192.

8. Antoniazza, G.; Nicollier, T.; Wyss, C.; Boss, S.; Rickenmann, D. Bedload Transport Monitoring in Alpine Rivers: Variability in Swiss Plate Geophone Response. Sensors 2020, 20, 4089. [CrossRef] [PubMed]

9. Wyss, C.; Rickenmann, D.; Fritschi, B.; Turowski, J.; Weitbrecht, V.; Travaglini, E.; Bardou, E.; Boes, R. Laboratory flume experiments with the Swiss plate geophone bed load monitoring system: 2 . Application to field sites with direct bed load samples. Water Resour. Res. 2016, 52, 7760-7778. [CrossRef]

10. Bombrun, L.; Vasile, G.; Gay, M.; Totir, F. Hierarchical segmentation of polarimetric SAR images using heterogeneous clutter models. IEEE Trans. Geosci. Remote Sens. 2011, 2, 726-737. [CrossRef]

11. Vasile, G.; d'Urso, G.; Charlatchka, R.; Lungu, E. Calibration of an Active Ultrasound Bedload Monitoring System for Underwater Environments. In Proceedings of the MTS/IEEE North American OCEANS Conference, Washington, DC, USA, 19-22 October 2015; pp. 1-4.

12. Vasile, G.; d’Urso, G.; Lungu, E. Transient Vibration Signal Analysis for Bedload Transport Monitoring Systems. In Proceedings of the MTS/IEEE North American OCEANS Conference, Anchorage, AK, USA, 18-21 September 2017; pp. 1-4.

13. Vasile, G.; Pascal, F.; Ovarlez, J.; Formont, P.; Gay, M. Optimal parameter estimation in heterogeneous clutter for high-resolution polarimetric SAR data. IEEE Geosci. Remote Sens. Lett. 2011, 8, 1046-1050. [CrossRef]

14. Conte, E.; DeMaio, A.; Ricci, G. Recursive estimation of the covariance matrix of a compound-Gaussian process and its application to adaptive CFAR detection. IEEE Trans. Image Process. 2002, 50, 1908-1915. [CrossRef]

15. Pascal, F.; Chitour, Y.; Ovarlez, J.P.; Forster, P.; Larzabal, P. Covariance structure maximum-likelihood estimates in compound Gaussian noise: Existence and algorithm analysis. IEEE Trans. Signal Process. 2008, 56, 34-48. [CrossRef]

16. Pascal, F.; Forster, P.; Ovarlez, J.P.; Larzabal, P. Performance analysis of covariance matrix estimates in impulsive noise. IEEE Trans. Signal Process. 2008, 56, 2206-2216. [CrossRef]

17. Salvador, S.; Chan, P. Determining the Number of Clusters/Segments in Hierarchical Clustering/Segmentation Algorithms. In Proceedings of the ICTAI 2004, 16th IEEE International Conference on Tools with Artificial Intelligence, Boca Raton, FL, USA, 15-17 November 2004; pp. 576-584. 